\title{
Application Of Spline Interpolation Filter In Strip Flatness Detection
}

\author{
Biao Cao ${ }^{1}$,ZhiQiang He ${ }^{1}$, Lin Yang ${ }^{2}$ LiXi Zhang ${ }^{1}$ \\ 1. Department of Basic Science,Naval Aeronautical University, Yantai 264001 China, Email:caobiao2001@126.com; \\ 2.Changan Power Research Institute, Chongqing 401120 China.
}

Keywords:vibrations; dolph-chebyshev window; piecewise cubic spline interpolation; flatness; fir low-pass filter.

\begin{abstract}
According to the analyzing of the vibrations in steel strips and its effect on flatness measurement based on the non-contact strip-flatness detection method, a vibration removing method is presented. The method firstly adopts FIR low-pass filter based on Dolph-Chebyshev Window function designing theory to remove the high frequency noise in the primitive flatness measurement data. As to consider the influence of random noise, there is additional noise message in low frequency range of flatness measurement data. Therefore, the method adopts piece-wise cubic spline interpolation to revise the data processed by low-pass filter. Application results show that the method have satisfied reliability, can effectively shorten the influence that the strip vibration caused on flatness measurement.
\end{abstract}

\section{Introduction}

Flatness is one of the most important features of rolled products ${ }^{[1]}$.But vibration will seriously affect the flatness detection result, especially in vision detection method $^{[4]}$.Therefor, it is significant to reduce the influence of vibration signal during strip-flatness detection.

The current flatness detection method can be divided into two types. One of detection methods based on hardware technology, such as Rometer ${ }^{[5]}$ multi-point detection method, $\mathrm{Xu}$ Hongzhe et $\mathrm{al}^{[3]}$ based on the image processing of the double Line detection method and Shapeline ${ }^{[5]}$ of the two-line detection method. All these methods used the principle of the distance invariance of two corresponding points to eliminate the effect of vibration, but it is poor in strip Non-vertical vibration types such as rotational vibration. The digital low-pass filter from software technology eliminates high-frequency interference in the flatness detection signal, but still contains noise information in the low-band flatness detection signal since the effect of random noise is not taken into account. For the lack of digital low-pass filter, Rubén Usamentiaga ${ }^{[4]}$ and others proposed the combination of the Butterworth IIR low-pass filter and RanSanc model estimation method. Although the Butterworth IIR low-pass filter eliminates the high frequency interference in flatness measurement of the strip, the stability of the filter is poor due to the inability to control the phase characteristics of the filter; and the RanSanc model estimation method is effective in processing the flatness measurement data Only in the inliers ${ }^{[6]}$ more than $30 \%$ of the case can be applied, otherwise it will affect the flatness detection robustness ${ }^{[4]}$. Therefore, in order to overcome the shortcomings of the above methods, a two-step strip vibration suppression method is proposed, which first uses the Dolph-Chebyshev Window ${ }^{[2]}$ FIR low-pass filter to filter the original data and then use the sub-cubic spline interpolation to correct the filtered data. Application result shows that the method can effectively filter the vibration signal of the strip on the flatness detection and improve the accuracy of flatness detection.

\section{Method of Strip-Flatness Measurement}

Due to the uneven distribution of internal residual stress after strip rolling, the longitudinal fiber strip of steel is unevenly extended, resulting in warping of the strip, that is flatness defects. Flatness defects include "edge waves", "waves", "single waves", "1/3 waves" and so on. According 
to the flatness detection methods' real-time performance, flatness detection can be divided into online detection and off-line detection of two categories.

Traditional flatness on-line detection technology using contact detection method such as ABB developed Stressmeter ${ }^{\circledR}{ }^{[5]}$, split roller tension detection technology $\mathrm{y}^{[5]}$ and so on, due to maintenance difficulties and easy to wear on the steel surface and gradually eliminated; other online Detection methods such as capacitor-based capacitive displacement sensing detection technology ${ }^{[4]}$ because of its high installation costs have been rarely used in the factory practice. Currently, the most mainstream flatness detection method is based on the laser triangulation principle of non-contact on-line detection method which uses three-dimensional reconstruction technology ${ }^{[7,8]}$, the measurement principle is simple, low cost, easy to maintain, is widely used in steel industry.

Figure 1 shows the schematic diagram of the on-line flatness detection system with direct beam laser. The laser emitter emits a structural light beam to the strip surface to form a laser spot, the plane array CCD sensor to collect the spot image. When the system running, the image acquisition module accurately collects the strip conveying speed according to the incremental rotary encoder installed in the system, and real-time image data is transmitted through the image acquisition card to the image processing module in the computer processing unit. The processing module successively image enhancement, image edge detection and spot center extraction of the spot image, and then transform the image coordinates of the center of the spot into the world coordinates of the spot on the strip surface using the three-dimensional reconstruction technique to measure the height of light spot relative to the reference plane. Finally, the length of the fiber strip $L^{j}$ at the measuring point $j(j=1$, $2,3,4,5)$ and the longitudinal fiber strip elongation $\varepsilon j^{[9]}$ at the measuring point $\mathrm{j}$ are obtained by integration.

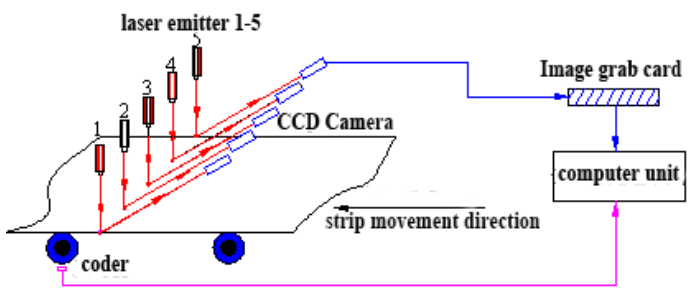

Fig.1 Schematic diagram of on-line flatness detection system

$$
\begin{gathered}
L^{j}=\sum_{i=0}^{N} \sqrt{\left(h_{i+1}^{j}-h_{i}^{j}\right)^{2}+\left(\frac{v_{i+1}+v_{i}}{2} \Delta t\right)^{2}} \\
\varepsilon_{j}=\frac{L^{j}-L^{r e f}}{L^{r e f}} \times 10^{5} \mathrm{I}
\end{gathered}
$$

Where $\mathrm{L}^{\text {ref }}$ is the average of the length of the measuring fiber strip; $v_{i}$ is the strip speed at time $I, \Delta t$ is the sampling period, $h_{i}^{j}$ is the height of the spot at the point $j$ on moment of point $\mathrm{i}$ relative to the reference plane; $\mathrm{N}$ is the sum of the number of sampling points, $I$ is the flatness unit.

Flatness Offline calibration results are usually validated as a result of flatness on-line test results. The principle of the test is to assume that the longitudinal fiber strip shape at the measuring point is approximately sinusoidal. By measuring the straight-line distance $L$ of the two adjacent peaks and the distance $\mathrm{H}$ from the crest to the trough, the flatness detection result is characterized by the flatness calibration index $\zeta_{j}^{[4]}$.

$$
\varsigma_{j}=\left(\frac{\pi H_{j}}{2 L_{j}}\right) \times 10^{5} \mathrm{I}
$$

Where $\mathrm{L}^{\mathrm{j}}$ is the peak-to-peak distance at point $\mathrm{j}$, and the distance from the peak to the trough is $\mathrm{H}_{\mathrm{j}}$.

\section{Effect of vibration on flatness measurement}

In the vibration-free condition, when the length of the longitudinal fiber strip on both sides of the strip is greater than the reference value $L^{\text {ref }}$, edge-waves will appear. when the length of the longitudinal fiber strip in the middle of the strip is greater than the reference value, central wave will appear, and the "single edge wave" will be produced when only one side of the strip's length is greater than the reference value. The Figure 2 shows the three-dimensional map of the flat surface of the strip with the wave, central wave and composite waves.(The width $\mathrm{W}=0: \mathrm{L}=110, \mathrm{H}=$ 4; $\mathrm{W}=100: \mathrm{H}=0=\mathrm{W}=200: \mathrm{L}=300, \mathrm{H}=3 ; \mathrm{W}=300: \mathrm{H}$ $=0 ; \mathrm{W}=400: \mathrm{L}=110, \mathrm{H}=4)$.

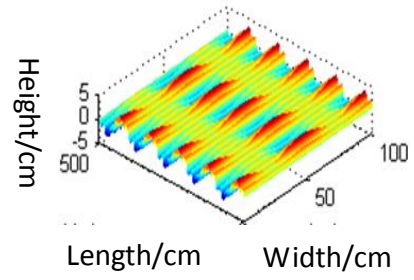

Fig.2 Steel strips with wavy edges and center buckle 

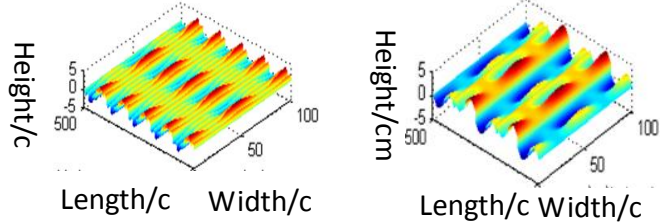

(a) Sinusoidal vibration ( $\mathrm{L}=5, \mathrm{H}=1)$ (b)Step vibration $(\mathrm{H}=1)$

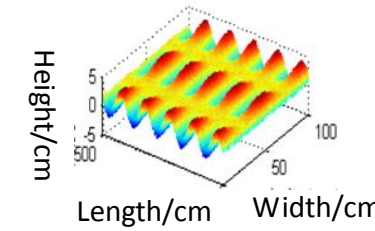

(c) random vibration $(\mathrm{H}=1)$

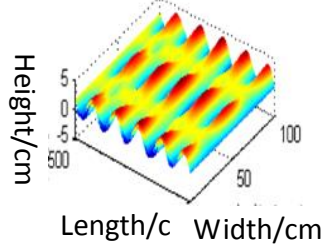

(d) $\operatorname{swivel}(\mathrm{L}=200, \mathrm{H}=2)$
Fig.3 3D-surface of strip steel with vibrations

In actual rolling process, due to the vibration of the strip factors, will make the strip in the roller on the cyclical or random movement. Figure 3 shows the three-dimensional surface of the strip with several typical vibration types on the basis of the composite wave in Fig. 2.

It can be seen from Fig. 3 that the strip steel will increase the length of the longitudinal fiber strip at the measuring point due to the vibration of the strip. and the three-dimensional surface of the strip changes sharply compared to Fig.2. And the vibration frequency at each point of the strip section is often different, resulting in the strip at the point of the longitudinal strip of fiber elongation ratio is not consistent, further caused the strip flatness test results of serious deviation, so it is necessary to effectively process the flatness measurement data and eliminate the interference information that affects flatness detection.

\section{Vibration signal suppression method}

\subsection{Dolph-chebyshev window FIR low-pass filter}

According to the time-domain characteristics of the impact response, the FIR filter or IIR filter can be selected when designing the digital low-pass filter. Compared to IIR filters, FIR filters are absolutely stable systems, while ensuring a strict linear phase, and the structure of the use of non-recursive structure is simple to design. The FIR filter based on the Dolph-Chebyshev Window function method has a filter characteristic parameter compared to the FIR filter designed with other window functions (such as rectangular window, Hamming window, Blackman window, Hamming window, etc.) Can ensure that the set Side-lobe height, adjust the moderate size of the main lobe, and the side lobe with the same ripple characteristics, the filter effect is better. Therefore, the Dolph-Chebyshev Window FIR low-pass filter is used to pretreat the strip flatness measurement signal, which can effectively filter out the high frequency signal in the flatness measurement signal of strip.

Let the ideal filter frequency response be $\mathrm{H}_{\mathrm{d}}\left(\mathrm{e}^{\mathrm{j} \omega}\right)$, and derive the infinite long time domain sequence $h_{d}(n)$ from the inverse Fourier transform:

$$
h_{d}(n)=\frac{1}{2 \pi} \int_{-\pi}^{\pi} H_{d}(n) e^{j n \omega} d \omega
$$

The time domain sequence $h_{d}(n)$ is truncated by the Chebyshev window function:

$$
h(n)=h_{d}(n) w(n)
$$

Where $h(n)$ is the time domain sequence after truncation, $w(n)$ is the Chebyshev window function.

$$
\begin{gathered}
w(n)=\frac{1}{2 M+1}\left[\frac{1}{r}+2 \sum_{k=1}^{M} T_{2 M}\left(\beta \cdot \cos \left(\frac{k \pi}{2 M+1}\right) \cdot \cos \left(\frac{2 n k \pi}{2 M+1}\right)\right)\right] \\
-M \leq n \leq M) \\
\beta=\cosh \left(\frac{1}{2 M} \operatorname{arccosh} \frac{1}{r}\right)
\end{gathered}
$$

Where $\mathrm{T}_{2 \mathrm{M}}(\mathrm{x})$ represents the Chebyshev polynomial, $r$ represents the Side-lobe relative amplitude, $M$ represents the window length.

FIR filter frequency response $\mathrm{H}\left(\mathrm{e}^{\mathrm{j} \omega}\right)$ by $\mathrm{h}(\mathrm{n})$ available:

$$
H\left(e^{j \omega}\right)=H_{d}\left(e^{j \omega}\right) \otimes W\left(e^{j \omega}\right)=\sum_{n=-M}^{M} h(n) \cdot e^{j n \omega}
$$

Where $\mathrm{W}\left(\mathrm{e}^{\mathrm{j} \omega}\right)$ is the amplitude response of the window function, Which has a direct effect on the performance of the filter.

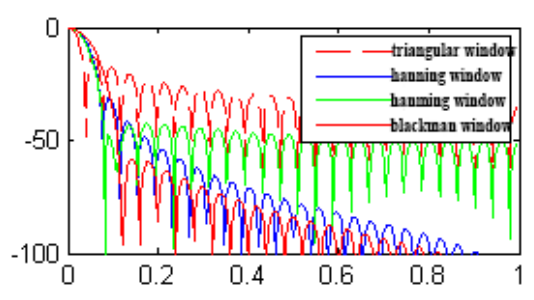

Fig.4 Comparision of amplitude response of several windows

From Figure 4, the amplitude response of several window functions (window length is 51 ), it can be seen from 
the rectangular window transition zone width is minimal but the stopband attenuation minimum, Blackman window stopband attenuation maximum but the transition band width maximum. The parameter of dolph-chebyshev window function is adjustable. fixed the stopband attenuation, the width of the main lobe decreases with the increase of the window length as shown in Fig. 5(b). fixed the length of the window, and the width of the main lobe are Large and larger as the stop-band attenuation increased, shown in Figure 5 (a). Therefore, when designing the filter, it is necessary to consider both the window length and stopband attenuation value to make FIR filter have better filtering characteristics.

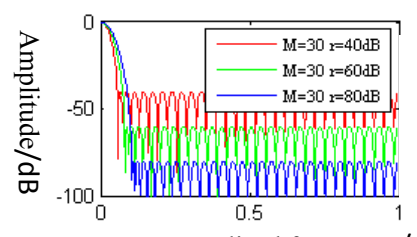

(a) Stopband attenuation
Normalized frequency/ $\pi$

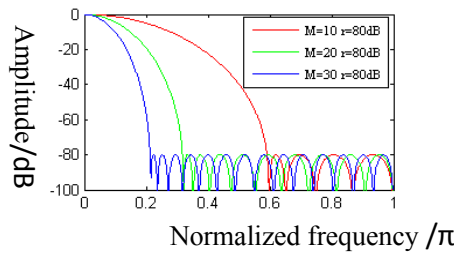

(b) window length
Fig.5 Amplitude response of dolph-chebyshev window

\subsection{Segmentation Cubic Spline Interpolation Compensation Algorithm}

Due to the influence of ambient noise and the vibration of the steel plate, the noise information is still included in the straightness measurement signal filtered by the dolph-chebyshev FIR low-pass filter. Rubén Usamentiaga ${ }^{[4]}$ proposed the use of RanSanc model estimation to compensate for the shortcomings of low-pass filter. The RanSanc model estimates the algorithm based on the set of samples containing the anomaly data to calculate the mathematical model parameters of the data to obtain a valid sample ${ }^{[6]}$, which is used to ensure that the correct data for the measurement exceeds $30 \%{ }^{[4]}$ and therefore the stability is poor. This paper propose a sub-cubic spline interpolation algorithm to compensate for the shortcoming of the filter. First of the algorithm is to find the approximate vibration balance position at a certain point by means of point-by-point scanning at the point height and then, interpolation. The algorithm is simple, stable and can effectively eliminate the low-band noise information.

Let $\left(x_{i}, y_{i}\right)$ represents the coordinates of the filtered extreme points, and the coordinates of the equilibrium point
$P_{j}\left(m_{j}, n_{j}\right)(j=1,2, \ldots, n-1) T$ represents the height threshold (only when the height value exceeds the set value), median means the median value.

$$
\begin{aligned}
& \text { when } \mathrm{y}_{\mathrm{i}}>\mathrm{T}, \\
& y_{i}=\operatorname{median}\left\{y_{i-2}, y_{i-1}, y_{i}, y_{i+1}, y_{i+2}\right\} \\
& p_{j}\left(m_{j}, n_{j}\right)=\left(\frac{x_{i+1}+x_{i}}{2}, \frac{y_{i+1}+y_{i}}{2}\right)
\end{aligned}
$$

According to the above method, find $(\mathrm{k}+1)$ equilibrium position points and have $\mathrm{m}_{0}<\mathrm{m}_{1}<\ldots .<\mathrm{m}_{\mathrm{k}}$, construct a cubic spline interpolation function $\mathrm{S}(\mathrm{x})$ satisfies the condition

1) $\mathrm{S}\left(\mathrm{x}_{\mathrm{i}}\right)=n_{i}(i=0,1,2, \ldots, k)$.

2) $\mathrm{S}\left(\mathrm{x}_{\mathrm{i}}\right)$ is cubic polynomial on $\left[\mathrm{m}_{\mathrm{i}}, \mathrm{m}_{\mathrm{i}+1}\right]$.

3) $\mathrm{S}\left(\mathrm{x}_{\mathrm{i}}\right)$ have second continuous derivatives on $\left[\mathrm{m}_{0}, \mathrm{~m}_{\mathrm{k}}\right]$. In the $\left[\mathrm{m}_{\mathrm{i}}, \mathrm{m}_{\mathrm{i}+1}\right](i=0,1,2, \ldots, k) \mathrm{S}\left(\mathrm{x}_{\mathrm{i}}\right)$ is:

$$
\begin{aligned}
& S(x)=\left(1+2 \frac{x-m_{i}}{m_{i+1}-m_{i}}\right)\left(\frac{x-m_{i+1}}{m_{i+1}-m_{i}}\right)^{2} n_{i}+\left(1+2 \frac{x-m_{i+1}}{m_{i}-m_{i+1}}\right)\left(\frac{x-m_{i}}{m_{i+1}-m_{i}}\right)^{2} n_{i+1} \\
& +\left(x-m_{i}\right)\left(\frac{x-m_{i+1}}{m_{i}-m_{i+1}}\right)^{2} s^{\prime}\left(x_{i}\right)+\left(x-m_{i+1}\right)\left(\frac{x-m_{i}}{m_{i+1}-m_{i}}\right)^{2} s^{\prime}\left(x_{i+1}\right)
\end{aligned}
$$

The unknown coefficient in Eq. (11) can be obtained from the boundary condition $\mathrm{S} "\left(\mathrm{~m}_{0}\right)=\mathrm{S} "\left(\mathrm{~m}_{\mathrm{k}}\right)=0$.

\section{Experiment of vibration filter}

Figure 6 shows the platform which was used to measure the vertical height of point 1 . The sampling frequency of $5000 \mathrm{~Hz}$, and collect 5000 sampling data points. Figure 7 and Figure 8, respectively, is the time-domain waveforms and spectrum analysis of point $1^{[10]}$.

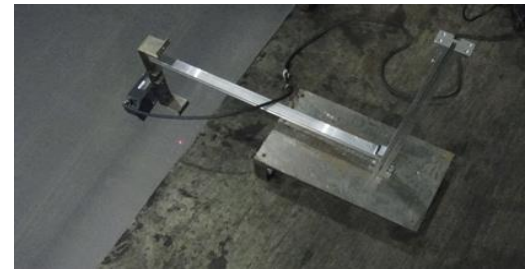

Fig.6 Flatness measurement in 1-dot

It can be seen from Fig. 6 that the height of the measuring point is always changing, and some high-frequency components are vibrating back and forth around the changing trend. The vibration is caused by the impact, raceway vibration and other factors, the amplitude of about $6 \mathrm{~mm}$. As can be seen from Fig.7, the frequency components causing the vibration are broad spectrum and low frequency. The low amplitude of the broad spectrum shows that the impact energy is small and the amplitude of 
the vibration is small too. The low frequency component contains the true displacement of the steel plate surface.

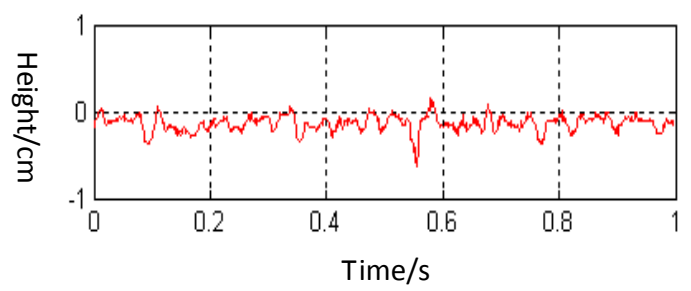

Fig.7 Time domain waveform

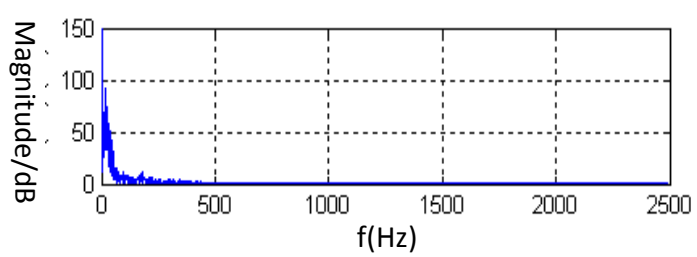

Fig.8 Frequency analysis

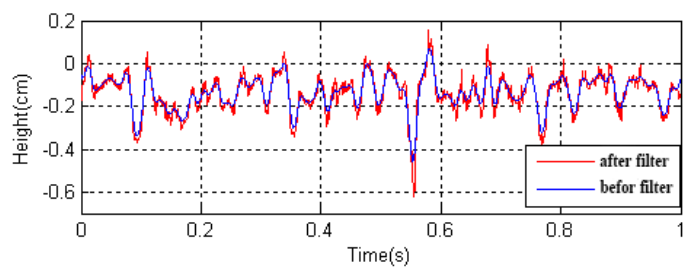

Fig.9 Comparision of time domain waveform

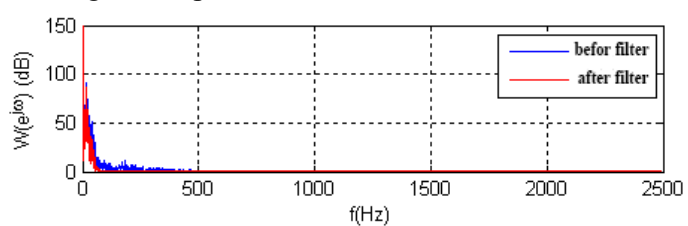

Fig.10 Comparison of frequency analysis

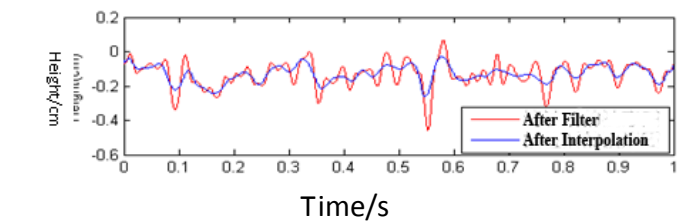

Fig.11 Comparison of time domain waveform between low-pass

filter and wise cubic spline interpolation

Set the filter stop-band attenuation of $80 \mathrm{~dB}$, Dolph Chebyshev window length 201, cut off frequency of $50 \mathrm{~Hz}$, the waveform before and after shown in Figure 9, the spectrum before and after filtering shown in Figure 10. It can be seen from Fig.10 that the high frequency portion exceeding $50 \mathrm{~Hz}$ is effectively filtered. Figure 10 shown that high-frequency interference has been filtered out, the wave is flat, but low frequency band noise information still exist. The results obtained by the sub-cubic spline interpolation compensation algorithm are shown in Fig.11. It can be seen from Fig.11 that the wave shape after treatment is smooth and reflect the real surface of the strip.

The flatness detection data at points 2 to 5 are successively processed by the above-described method. In order to verify the above test results, the detection values and off-line calibration results of comparative analysis as shown in Table 1 below. It can be seen from the table that the relative error between the interpolation filter processing data and the flatness detection result obtained by off-line calibration is not more than $6 \%$. It is proved that this method can effectively filter the influence of strip vibration on flatness detection.

\section{Conclusion}

In this paper, by analyzing the effect of strip vibration on the flatness detection, a method of vibration suppression of strip is proposed, which is based on the design of the FIR-low-pass filter using the Dolph-Chebyshev window function, and then use the sub-cubic spline interpolation compensation algorithm to eliminate the inclusion of noise in the low-frequency information. The experimental results show that the relative error between the flatness detection result and the off-line calibration result is less than $6 \%$. 


\begin{tabular}{ccccc}
\hline $\begin{array}{c}\text { Test } \\
\text { point }\end{array}$ & $\begin{array}{c}\text { Calculated flatness by } \\
\text { Dolph-chebwin low-pass } \\
\text { filter (I) }\end{array}$ & $\begin{array}{c}\text { The calculated flatness by this paper's } \\
\text { method (I) }\end{array}$ & $\begin{array}{c}\text { The flatness by } \\
\text { off-line calibration } \\
(\mathrm{I})\end{array}$ & $\begin{array}{c}\text { Relative } \\
\text { error } \\
(\%)\end{array}$ \\
\hline 1 & 133.518 & 10.762 & 11.264 & -4.46 \\
2 & 66.667 & 8.445 & 8.938 & -5.52 \\
3 & 226.533 & 28.126 & 27.034 & 4.04 \\
4 & 141.016 & 12.883 & 13.643 & -5.57 \\
5 & 520.519 & 58.776 & 56.326 & 4.35 \\
\hline
\end{tabular}

Table.1 Result analysis of flatness calculation

\section{References}

[1] Rubén Usamentiaga, Julio Molleda, Daniel F. García ,etal. Mechine Vision System for Flatness Control Feedback. 2009 Second International Conference on Machine Vision. Dubai, 2009,USA: IEEE, 2009:105-110.

[2] Peter Lynch.The Dolph-Chebyshev Window: A Simple Optical Filter [J]. Monthly Weather Review,1997, 125:655-657.

[3] Xu Hongzhe, Liu Kai, Peng Xiaohui, et al. Reaserch of the image processing in dynamic flatness detection based on improved laser triangular method[J]. Academic Journal of $\mathrm{Xi}$ ' an Jiaotong University. 2008,20(3):168-171.

[4] Rubén Usamentiaga, Julio Molleda, Daniel F. García ,et al. Vibration in steel strips: effect on flatness measurement and filtering. Industry Applications Society Annual Meeting. Lake Buena Visata, 2013,USA:IEEE,2013:1-10

[5] Molleda J, Usamentiaga R, García DF. On-Line Flatness Measurment in the Steelmaking Industry[J]. Sensors, 2013, 13(8):10245-10272.

[6] M. Fischler, R. Bolles. Random sample consensus: a paradigm for model fitting with application to image analysis and automated cartograph[J]. Communication of ACM, 1981,24(6):381-384.

[7] F. Pernkopf. 3d surface acquisition and reconstruction for inspection of raw steel products[J]. Computer in Industry, 2005,56(8):879-884.

[8] Rubén Usamentiaga, Julio Molleda, Daniel F. García. Shape Measurment of Steel Strip Using a Laser-Based Three-Dimentinal Reconstruction Technique[J]. IEEE transaction on Industry applcation.2011, 47(4):1539-1540.

[9] Yang Xilin, Qiu Zhongyi, Yu Changyou ,et al. Study on Laser Shape Measurement System for Hot-Rolling Strip Steel. Chiba,1998, USA: IEEE, 1998:911-912.

[10] Ma Jiangcang, Shi Qingbin, Cheng cunhu,et al. VibrationSignal Seperation Thechniques in Aeroengine Test[J]. Journal of Vibration Measurement \& Diagnosis,2009,29(1):3-4. 